\title{
Will solid hydrogen ever be a metal?
}

\section{Peter P. Edwards and Friedrich Hensel}

At ultra-high pressures, liquid hydrogen becomes metallic. So should solid hydrogen, yet it stubbornly resists. A newly predicted spontaneous asymmetry of molecules in the solid may be the reason.

n

n 1926 J. D. Bernal proposed that all matter, when subjected to a high enough pressure, will inevitably become metallic - that is, it will be permeated by a sea of completely free electrons that conduct electricity easily. The most enticing substance for pressure-induced metallization is, in fact, the lightest and supposedly the simplest of all the elements in the periodic table - hydrogen. Wigner and Huntington in 1935 first predicted that molecular diatomic hydrogen would undergo a transition to a metallic state at an imposed pressure of about 250,000 atmospheres ${ }^{1}$; current predictions are in a range close to three million atmospheres. But despite an unrelenting experimental assault at these ultra-high pressures, dense solid hydrogen has so far defied all attempts at metallization ${ }^{2}$. On page 652 of this issue ${ }^{3}$, Edwards and Ashcroft describe a new twist to the continuing saga, which may help explain the recalcitrance of solid hydrogen towards metallization.

Why are we so interested in making metallic hydrogen? It may have exotic properties and important applications. For example, it has been predicted to be a roomtemperature superconductor; and if it could be stably quenched from high pressures to ambient, it would be a highly effective and exceptionally clean propellant for space travel. The metallization of hydrogen would also provide new insights into the nature of giant planets - Jupiter and Saturn, for example, both contain more than 400 Earth masses, most of which is hydrogen under extreme conditions.

Edwards and Ashcroft have discovered a remarkable spontaneous electronic polarization of hydrogen dimer molecules at ultra-high pressures. In stark contrast to the room-pressure situation, in which a hydrogen molecule has precisely equivalent electron (charge) density at both protons, at high pressures the electronic charge piles up preferentially at just one of the constituent protons. The hydrogen molecule thus develops a permanent electric dipole moment. This induced ionic character - in the limit one would regard hydrogen as 'protonium hydride, $\mathrm{H}^{+} \mathrm{H}^{-}$- may serve to delay, or even thwart completely, the long-awaited transition to the metallic state of dense solid hydrogen.
The pressure-induced metallization of solid hydrogen can be viewed in terms of electronic band theory. At low pressures the solid is an infinite crystalline assembly of isolated $\mathrm{H}_{2}$ molecules, all weakly interacting. The electrons are all bound to their molecules, and need to be freed to conduct electricity: in band-theory terms, we have a completely filled valence band separated by a very large electronic energy gap $(>13 \mathrm{eV})$ from the empty conduction band (Fig. 1). Under ambient pressure, elemental solid hydrogen could only conduct by the thermal excitation of large numbers of electrons from the valence band to the conduction band. That would require enormously high temperatures.

Solid hydrogen is thus an insulator. But as the applied pressure increases, $\mathrm{H}_{2}$ molecules begin to interact more strongly, and the energy bands widen. In the limit of zero band gap we would have an insulator-to-metal transition - and metallic hydrogen.

Several groups have already succeeded in compressing solid hydrogen to ultra-high pressures, with an elemental density now more than 10 times the room-pressure solid density $^{2,4}$. The average density of electrons in dense solid hydrogen is now two to three times that of the excellent metal, aluminium. And yet, remarkably, solid hydrogen under these crushingly oppressive conditions remains a stubborn insulator, with not even a hint of metallic behaviour at low tempera-

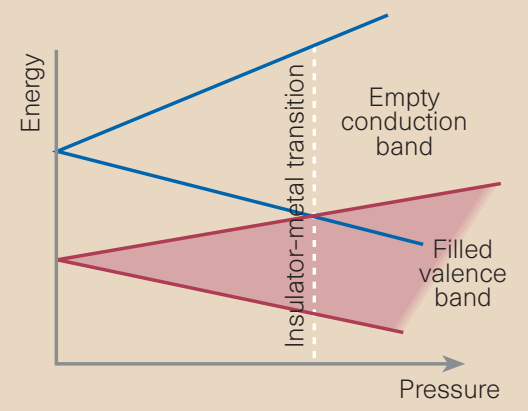

Figure 1 Bands under pressure. With increasing pressure, and therefore density, the electronic energy bands of solid hydrogen broaden. One assumes that eventually they will overlap, making a conducting, metallic state; but spontaneous polarization of the $\mathrm{H}_{2}$ molecules (Fig. 3) might postpone that, or even prohibit it. tures. The consequences of squeezing solid hydrogen are clearly a good deal more subtle than at first thought, and it turns out that this, the 'simplest' of all molecular solids, has a rich and remarkably complex phase diagram $^{2,4,5}$ (Fig. 2).

In phase I of solid hydrogen, the so-called orientationally disordered state, the individual molecules execute complete rotational motion in addition to the usual molecular vibrations.

Below a temperature of about $120 \mathrm{~K}$, solid hydrogen undergoes a transition at about 1.5 million atmospheres to phase II, a state in which the constituent $\mathrm{H}_{2}$ molecules become 'frozen' in a random orientation in the crystal.

Phase III is undoubtedly the most intriguing, because elemental hydrogen remains in this state up to the highest pressures yet achieved, and still does not become metallic. Perhaps the most remarkable thing about phase III is the appearance of a striking absorption of infrared radiation, which is completely absent in the isolated molecule and largely so in the solid at lower pressures (phases I and II).

Why is the observation of infrared activity in phase III of dense hydrogen particularly important? Substantial infrared activity can only arise from molecules with an intrinsic electric dipole moment. But free $\mathrm{H}_{2}$ molecules have a spatially symmetric electron density distribution between their two protons, and so do not possess an intrinsic dipole moment. All hydrogen molecules should therefore show little, if any, infrared activity in the dense solid. However, in 1994 strong infrared activity was detected in solid hydrogen at low temperatures and high pressures, occurring only in phase III. Importantly,

this fascinating behaviour occurs under conditions for which solid hydrogen still shows no evidence of metallic behaviour.

Under ambient pressure, states of $\mathrm{H}_{2}$ that have any dipolar character are much higher in energy than the unpolarized states. But Edwards and Ashcroft ${ }^{3}$ note that, as the band

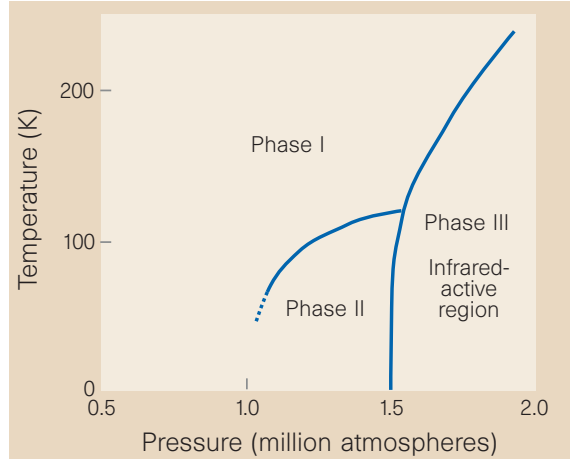

Figure $2 \mathrm{~A}$ possible phase diagram for dense hydrogen. Phase III, which absorbs infrared light strongly, may be characterized by a spontaneous polarization of the constituent hydrogen molecules. 


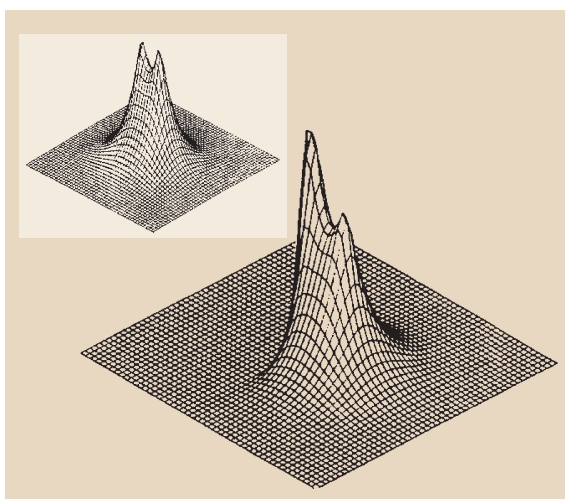

Figure 3 The newly predicted ground state of molecular hydrogen at ultra-high pressure ${ }^{3}$. Here the electron (charge) cloud preferentially accumulates at just one of the two protons, producing an electric dipole. (The magnitude of this charge transfer has been exaggerated for clarity.) The inset shows molecular hydrogen under normal conditions; here the two protons are surrounded by a symmetrical charge cloud.

gap of solid hydrogen closes continuously with pressure, there comes a point at which it may be energetically favourable to mix in a small proportion of these ionic states, resulting in a hybridized ground state. The pressure-induced electric dipole formed on any one of these hydrogen molecules will interact with, and be stabilized by, the other remaining molecules in the dense solid. The stabilization of this new dipolar state will be pressure dependent, and so above a critical density a spontaneous permanent electric polarization sets in on each molecule (Fig. 3).

Edwards and Ashcroft calculate the necessary conditions for the evolution of an induced molecular dipole in hydrogen. They predict the appearance of a spontaneous electronic polarization at a pressure where, experimentally, one sees the onset of the striking infrared activity in hydrogen. So dense hydrogen in phase III is composed of molecular, dipolar hydrogen. There may also be a reorientation and displacement of the dipolar molecules as the new, partly ionic state of hydrogen forms.

But what of the anticipated transition to metallic hydrogen at even higher densities? It has been suggested that molecular hydrogen may eventually become fully ionic, namely $\mathrm{H}^{+} \mathrm{H}^{-}$, with enough compression ${ }^{6}$. But from their calculations Edwards and Ashcroft find that the system does not seem to be progressing towards a fully ionic state.

What seems clear, however, is that the presence of even partially ionic character in the ground state of dense solid hydrogen will act to widen the previously narrowing band gap and hence frustrate the transition to the long-sought metallic state. Will solid hydrogen ever become a metal? A pessimistic prospect, contrary to Bernal's optimistic 1926 generalization, might be that solid hydrogen may never achieve metallic status.
Only time - and pressure - will tell.

Peter P. Edwards is in the School of Chemistry, The University of Birmingham, Edgbaston, Birmingham B15 2TT, UK. Friedrich Hensel is in the Fachbereich Chemie, Philipps-Universität Marburg, D-35032 Marburg, Germany.

\footnotetext{
1. Wigner, E. \& Huntington, H. B. J. Chem. Phys. 3, 764-770 (1935).

2. Silvera, I. F. in Metal-Insulator Transitions Revisited (eds

Edwards, P. P. \& Rao, C. N. R.) 21-42 (Taylor \& Francis, London, 1995).

3. Edwards, B. \& Ashcroft, N. W. Nature 388, 652-655 (1997).

4. Mao, H.-K. \& Hemley, R. J. Rev. Mod. Phys. 66, 671-692 (1994).

5. Ashcroft, N. W. Phys. World 8, 43-47 (1995).
}

\section{Even-toed fingerprints on whale ancestry}

\section{Michel C. Milinkovitch and J. G. M. Thewissen}

$\sum$ th morphological ${ }^{1}$ and molecular ${ }^{2}$ studies indicate that cetaceans (whales, dolphins and porpoises) and artiodactyls (even-toed ungulates, which include pigs, hippos, camels and ruminants) form a clade or monophyletic group - that is, they have a common ancestor that is not shared by any other group of mammals. This is counter-intuitive, because it implies that a cow is more closely related to a dolphin or a whale than to a horse, yet it is one of the best examples of congruence between morphological and molecular estimates of mammalian phylogeny.

The molecular analyses of Shimamura et $a l^{3}$, reported on page 666 of this issue, further disrupt phylogenetic dogma. Indeed, not only do the authors confirm the close relationship between artiodactyls and cetaceans, but they propose that cetaceans are deeply nested within the phylogenetic tree of the artiodactyls. These results strikingly contradict the common interpretation of the available morphological data (sup- porting artiodactyl monophyly) and, if correct, would make a cow or a hippopotamus more closely related to a dolphin or a whale than to a pig or a camel (Fig. 1).

Although it is compatible with earlier molecular analyses (for example, refs 4, 5), the idea that cetaceans are highly derived artiodactyls was first suggested in 1994 on the basis of mitochondrial and nuclear DNA and amino-acid sequences ${ }^{6}$. The idea was corroborated by other phylogenetic analyses of DNA sequences ${ }^{7}$. But the issue is still controversial, because the exact means by which molecular sequence data should be analysed remains debated - although analytical settings that are particularly meaningful with respect to phylogenetic inferences can probably be identified in specific instances ${ }^{8}$. But, basically, many morphologists consider that molecular data are necessarily more noisy than morphological data.

The analyses by Shimamura and colleagues now provide a remarkable example of molecular markers, which should lead
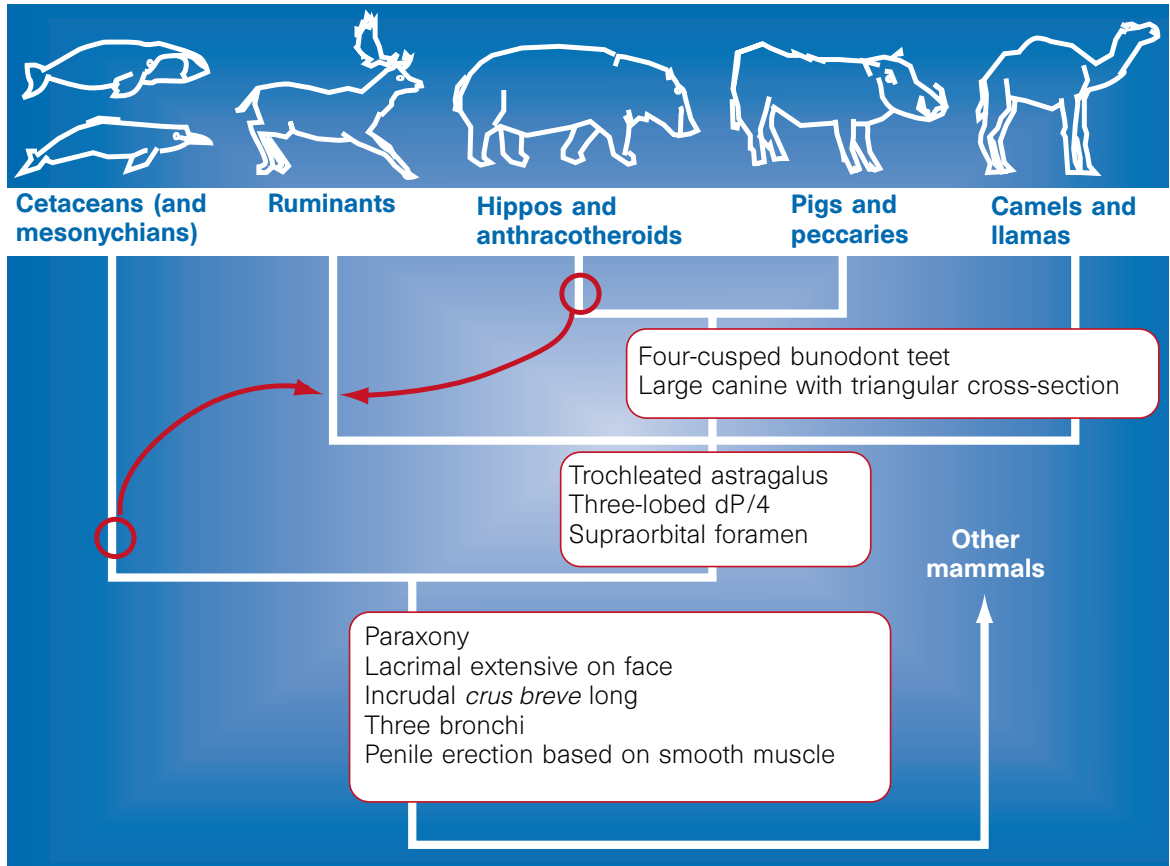

Figure 1 Twisted or untied? Shimamura et al. ${ }^{3}$ propose to attach the lineages of hippos and cetaceans (curved arrows, red) to the ruminant branch on this phylogenetic tree of artiodactyls - a marked diversion from the traditional view (white branching pattern). The two smaller boxes summarize some of the morphological evidence that disagrees with the new data. 